\title{
Continuous and discrete models in the mechanics of deformable solid bodies
}

\author{
Géza Lámer \\ University of Debrecen, Faculty of Engineering, Department of Engineering Management and Enterprise, 4028 Debrecen, Ótemető u. 2- \\ 4., Hungary
}

\begin{abstract}
The study provides an overview of modelling possibilities for the mechanical behaviour of media. The discrete, continuous or differential geometric as well as the discrete nature and continuous description grid continuum model in particular are highlighted. We point out that the differential geometric model is based on the concept of continuity and interprets a continuous medium model. We reveal that the grid continuum model is based on the application of numerical method and interprets a discrete medium model.
\end{abstract}

\section{Introduction}

The system of discretely located elements, classical continuum and generalised continuum can be used in literature to describe the mechanical behaviour of deformable solid bodies. The discrete system can be characterised as describe the system in both physics space and phase space as discrete domain functions. The classical continuum can be characterised by interpreting it as a continuous geometric locus in the physical space, applying continuous functions in the phase space to describe its condition. The generalised continuums can be described by modelling a discrete geometric locus retaining the internal structure of the matter in the physical space (e.g. with ,micro-continuums" sitting on the grid points of some kind of grid system), we characterise the condition of the internal structure matter with continuous functions for the phase space description.

The continuity of the three models differs in physical and phase space. We examine the three models according to this distinction in this study.

\section{Characterisation of discrete modelling in physical space}

\subsection{Description of the discrete model}

There are the bodies, degrees of kinematic freedom of bodies, methods of interaction existing among bodies, dynamic degrees of freedom, matter equations, the conditions of equilibrium, and the relationships describing the equilibrium (movement), the initial positions of the bodies and the initial values of dynamic effects in the initial positions, those dynamic, occasionally kinematic conditions, which exist when we seek the equilibrium of the system and its movement.
The system can be examined limited to mass points and rigid body sitting in grid points (it can be demonstrated that its deformability does not play any role), solution for the system can be examined without actually applying equilibrium (movement) equations. It is enough to limit ourselves to what degree of kinematic freedom does an element sitting on the grid points possesses and what equilibrium (movement) equations can be applied.

Hereinafter, the equation system of the discrete mechanic system is formally described. Provided there are $n$ numbers of elements. The location of elements (reference points) is marked with $\mathbf{r}_{i}$, the position of elements (basis vectors of tangential space) is marked with $\boldsymbol{\psi}_{i}$. Elements can have displacement $\mathbf{u}_{i}$ and rotation $\boldsymbol{\varphi}_{\mathrm{i}},(i$ $=1,2,3 \ldots n)$ degrees of freedom. The case of two degrees of freedom is examined separately and jointly as well.

Case of displacement: The internal force between the $i^{\text {th }}$ and $j^{\text {th }}$ element, $\mathbf{F}_{i j}$ and $\mathbf{F}_{j i}=-\mathbf{F}_{i j}$, depends on the distance of the two elements. The distance of the two elements is marked with $\mathbf{r}_{i j}=\mathbf{r}_{i}-\mathbf{r}_{j}$ in the initial state. This vector changes to value $\mathbf{R}_{i j}$ with the shift of the two elements, where $\mathbf{R}_{i j}=\mathbf{r}_{i j}+\mathbf{u}_{i}-\mathbf{u}_{j}$. Accordingly, the following forces act upon the $i^{\text {th }}$ element:

$$
\begin{aligned}
\mathbf{F}_{i j}=\mathbf{F}_{i j}\left(c_{i j}, \mathbf{r}_{i j}, \mathbf{u}_{i}, \mathbf{u}_{j}\right), & \left(i=1,2,3 \ldots n, j=1,2,3 \ldots m_{i} ; m_{i} \leq n\right) .
\end{aligned}
$$

Here, the $m_{i} \leq n$ inequality refers to the fact that there is not always a relationship between all elements in fact, different elements can have a relationship with a different number of elements. The fact that the magnitude of force between the $i^{\text {th }}$ and the $j^{\text {th }}$ elements can depend on various parameters was marked with $c_{i j}$ in the context regarding $\mathbf{F}_{i j}$ force. The equilibrium of the system for every $i^{\text {th }}$ element is given by force equations:

$$
\sum_{j=1}^{m_{i}} \mathbf{F}_{i j}\left(c_{i j}, \mathbf{r}_{i j}, \mathbf{u}_{i}, \mathbf{u}_{j}\right)+\mathbf{P}_{i}=\mathbf{0}, \quad(i=1,2,3 \ldots n)
$$

\footnotetext{
* Corresponding author: glamer@eng.unideb.hu
} 
The $\mathbf{P}_{i}$ in the equation is the ,external" force acting upon the $i^{\text {th }}$ element.

It can be seen from the equation system (we are in a three-dimensional space), that $3 n$ equations are available for determining $3 n$ variables. Starting from the known $\mathbf{r}_{i}$ positions of the individual elements in the task, the $\mathbf{R}_{i}=\mathbf{r}_{i}$ $+\mathbf{u}_{i}(i=1,2,3 \ldots n)$ is the new position of elements that can be determined under the effect of external forces: $\mathbf{P}_{i}$ $(i=1,2,3 \ldots n)$.

Case of rotation: The internal torque between the $i^{\text {th }}$ and $j^{\text {th }}$ elements, $\mathbf{M}_{i j}$ and $\mathbf{M}_{j i}=-\mathbf{M}_{i j}$, depends on the relative position of the two elements. The relative position of the two elements is marked with $\boldsymbol{\psi}_{i j}=\boldsymbol{\psi}_{i}-\boldsymbol{\psi}_{j}$ in the initial state. This vector changes to $\boldsymbol{\Psi}_{i j}$ value with the rotation of the two elements, where $\boldsymbol{\Psi}_{i j}=\boldsymbol{\psi}_{i j}+\boldsymbol{\varphi}_{i}-\boldsymbol{\varphi}_{j}$. Accordingly, the following torques act upon the $i^{\text {th }}$ element:

$$
\begin{aligned}
\mathbf{M}_{i j}= & \mathbf{M}_{i j}\left(d_{i j}, \boldsymbol{\Psi}_{i j}, \boldsymbol{\varphi}_{i}, \boldsymbol{\varphi}_{j}\right), \\
& \left(i=1,2,3 \ldots n, j=1,2,3 \ldots m_{i} ; m_{i} \leq n\right) .
\end{aligned}
$$

See the annotation regarding the $m_{i} \leq n$ inequality above. The fact that the magnitude of torque between the $i^{\text {th }}$ and $j^{\text {th }}$ elements can depend on various parameters was marked with $d_{i j}$ in the context regarding the $\mathbf{M}_{i j}$ torque. The equilibrium of the system for every $i^{\text {th }}$ element is given by torque equations:

$$
\sum_{j=1}^{m_{i}} \mathbf{M}_{i j}\left(d_{i j}, \boldsymbol{\Psi}_{i j}, \boldsymbol{\varphi}_{i}, \boldsymbol{\varphi}_{j}\right)+\mathbf{M}_{i}=\mathbf{0}, \quad(i=1,2,3 \ldots n) .
$$

The $\mathbf{M}_{i}$ in the equation is the ,external” torque acting upon the $i^{\text {th }}$ element.

It can be seen from the equation system that $3 n$ equations are available for determining $3 n$ variables. Starting from the known $\boldsymbol{\psi}_{i}$ position of the individual elements in the task, the $\boldsymbol{\Psi}_{i}=\boldsymbol{\psi}_{i}+\boldsymbol{\varphi}_{i}(i=1,2,3 \ldots n)$ is the new position of elements that can be determined under the effect of external torques: $\mathbf{M}_{i}(i=1,2,3 \ldots n)$.

Case of displacement and rotation: The internal force between the $i^{\text {th }}$ and $j^{\text {th }}$ elements, internal torque, depends on the distance and relative position of the two elements, see above. Accordingly, the following forces and torques act upon the $i^{\text {th }}$ element:

$$
\begin{gathered}
\mathbf{F}_{i j}=\mathbf{F}_{i j}\left(c_{i j}, \mathbf{r}_{i j}, \mathbf{u}_{i}, \mathbf{u}_{j}, d_{i j}, \boldsymbol{\Psi}_{i j}, \boldsymbol{\varphi}_{i}, \boldsymbol{\varphi}_{j}\right), \\
\mathbf{M}_{i j}=\mathbf{M}_{i j}\left(c_{i j}, \mathbf{r}_{i j}, \mathbf{u}_{i}, \mathbf{u}_{j}, d_{i j}, \boldsymbol{\Psi}_{i j}, \boldsymbol{\varphi}_{i}, \boldsymbol{\varphi}_{j}\right), \\
\quad\left(i=1,2,3 \ldots n, j=1,2,3 \ldots m_{i} ; m_{i} \leq n\right) .
\end{gathered}
$$

See the annotations regarding the $m_{i} \leq n$ inequality and $c_{i j}$ and $d_{i j}$ above. The equilibrium of the system for every $i^{\text {th }}$ element is given by force and torque equations:

$$
\begin{gathered}
\sum_{j=1}^{m_{i}} \mathbf{F}_{i j}\left(c_{i j}, \mathbf{r}_{i j}, \mathbf{u}_{i}, \mathbf{u}_{j}, d_{i j}, \boldsymbol{\Psi}_{i j}, \boldsymbol{\varphi}_{i}, \boldsymbol{\varphi}_{j}\right)+\mathbf{P}_{i}=\mathbf{0}, \\
\sum_{j=1}^{m_{i}} \mathbf{M}_{i j}\left(c_{i j}, \mathbf{r}_{i j}, \mathbf{u}_{i}, \mathbf{u}_{j}, d_{i j}, \boldsymbol{\Psi}_{i j}, \boldsymbol{\varphi}_{i}, \boldsymbol{\varphi}_{j}\right)+\mathbf{M}_{i}=\mathbf{0},
\end{gathered}
$$

where $(i=1,2,3 \ldots n)$.
The $\mathbf{P}_{i}$ in the equation is the ,external” force acting upon the $i^{\text {th }}$ element, while the $\mathbf{M}_{i}$ is the ,external" torque acting upon the $i^{\text {th }}$ element.

It can be seen from the equation system that $2 \times 3 n$ equations are available for determining $2 \times 3 n$ variables. Starting from the known $\mathbf{r}_{i}$ position and $\boldsymbol{\psi}_{i}$ direction of the individual elements in the task, the new position $\mathbf{R}_{i}=\mathbf{r}_{i}+$ $\mathbf{u}_{i}$, and its new direction $\boldsymbol{\Psi}_{i}=\boldsymbol{\psi}_{i}+\boldsymbol{\varphi}_{i}$ of elements $(i=1,2,3$ $\ldots n$ ) can be determined under the effect of $\mathbf{P}_{i}$ external forces and $\mathbf{M}_{i}$ external torques $(i=1,2,3 \ldots n)$.

\subsection{Possibilities and limitations of the discrete model}

The discrete method provides an opportunity to examine the mechanical condition of a system consisting of a finite number of mass points or rigid bodies with assumption that the mass point has three displacement, and the rigid body has three displacement and three rotation degrees of freedom, the known position vectors and the direction of the individual rigid bodies enables the determination of force between two-two mass points and the determination of force and torque between two-two rigid bodies. These internal forces depend on the displacement of mass points, and the internal forces and torques depend on the displacement and rotation of rigid bodies. The equations of equilibrium (movement) of mass point and rigid body provide the same number of equations as the number of kinematic variables. The solution of equilibrium equations provides the locations of mass points and the location and direction of rigid bodies depending on external forces, or external forces and torques.

The system provides a solution regarding the discrete points of space. That means that the theory for unknown quantities (as functions) whether they are displacements, rotations, forces or torques can only determine values in the discrete points of the physical space.

\section{Continuous in physical space model}

\subsection{Description of continuous in physical space model}

A body is given as a continuous model, along with its location in space, position, shape, characteristics of material behaviour, support conditions of the body, effects on the body, kinematic and dynamic values in the initial position of the body.

We identify a deformable body in the continuous model with its region occupied in the Euclidean space. Let there be given the $\left\{q^{i}\right\}$ coordinate system. The position vector is marked with $\mathbf{r}$ before the deformation, and with an $\mathbf{R}$ after the deformation.

Firstly, overview the quantities describing the geometry; see the relationships themselves e.g. [1,2].

- Basis vectors in the $\mathbf{r}$ position vector point.

- Components of the metric tensor.

- Partial differentials of basis vectors.

- Affine coefficients (Christoffel symbols).

- Christoffel-Riemann curvature tensor for expressing the curvature of the Riemann space. 
The following geometric terms can be expressed with the abovementioned quantities:

- scalar products of vectors $\mathbf{a}$ and $\mathbf{b}$,

- angles between vectors $\mathbf{a}$ and $\mathbf{b}$ (cosine),

- regular arc length between two points in space,

- absolute differentials,

- parallel shift along the curve.

The basis of a continuous model is the continuity itself. This enables the application of Taylor Series, which is applied in practice in the following form:

$$
F(x+\Delta x, y, z)=F(x, y, z)+\{\partial F(x, y, z) / \partial x\} \Delta x .
$$

The consequence of this is that differential relationships are established among the unknown quantities.

According to the continuous model, the position vector of all points of the deformable rigid body changes under external effect. In accordance with this, the differential geometric description of space can be used for the kinematic characterisation of the body. The interpreted terms are the following [1,2].

The difference of position vectors is the displacement vector: $\mathbf{u}=\mathbf{R}-\mathbf{r}$, this also describes the changes of the coordinate system at the same time. The components of the metric tensor in the deformed state are as the scalar product of basis vectors. The difference of the metric tensors can be relating to the deformation. The difference of the two tensors is the measurement tensor of the deformation: $\gamma=\mathbf{G}-\mathbf{g}$. The relative stretch of a direction vector is described by the main diagonal elements, the change in the angle between two direction vectors is described by the off-diagonal elements of the measurement tensor [2], when the relative strains (stretches and angel changes) are small [3-5]. This leads to the tensor of small strains: $\boldsymbol{\varepsilon}=(\mathbf{G}-\mathbf{g}) / 2$. The change of affine connexion coefficient, $\lambda_{i j}^{k}=\Gamma_{i j}^{k}-\Gamma_{i j}^{k}$, the relationships required for the equilibrium equations of the deformed state. The change of the curvature tensor provides the compatibility equations (if we make it equal to zero). The expression of different geometric objects with the components of the gradient tensor of displacement vector is required for establishing the theory. Here, we only provide a relationship for one geometric object regarding the measurement tensor of the strain: $\quad \gamma=\nabla \mathbf{u}+(\nabla \mathbf{u})^{*}+(\nabla \mathbf{u})(\nabla \mathbf{u})^{*} . \quad$ The other relationship can also be expressed with the gradient tensor of the displacement, see e.g. [1-5].

By describing deformation with displacement two further characterisations of changes in space can be provided. The rigid body rotation of the neighbourhood of a points (the tangential basis vectors to the point) is given by orthogonal component of the polar decomposition of the gradient tensor of the deformed position vector. The other is the rotation of a direction. This is provided by the whole gradient tensor of the deformed position vector.

The displacement of the points of the continuum clearly determines the rotation of neighbourhood of all the points. This cannot be an independent quantity. Con- sequently, neither the rotation of a direction, nor the rotation of the neighbourhood of a point can be interpreted as an independent kinematic variable.

The kinematic characterisation of the continuum can be summarised as follows. The primary kinematic variable of the continuum is the displacement field, while the secondary kinematic variable is the symmetrical strain tensor. The displacement of the continuum per points clearly provides the relative stretch and rotation of every unit vector, the angle change per point between two-two unit vectors and the rigid-body like rotation of the neighbourhood of every point (that is to say the tangential basis vectors belonging to the point).

Note: We have $3+6$ kinematic variables, with six strain-displacement relationships (the kinematic equations). The kinematic description of the continuum indefinite.

We wish to interpret internal forces in the continuous model in physical space as continuous. As a point has no dimension, no weight and only one concentrated force can be assigned to it in the physical space, the Newtonian model has to be modified so that we assign dynamic quantity to a small but finite volume rather than to a point. Avoiding details, let there be $\boldsymbol{\sigma}_{\mathbf{n}}(x, y, z)$ vector quantity on an surface with $\mathbf{n}$ outward normal, which if we sum (integrate) on finite size surface figure, then ultimately we obtain concentrated force (force vector):

$$
\int_{\Delta A} \boldsymbol{\sigma}_{\mathbf{n}}(x, y, z) d A=\mathbf{F}_{\mathbf{n}, \Delta A}(x, y, z) .
$$

The $\boldsymbol{\sigma}_{\mathbf{n}}(x, y, z)$ quantity introduced this way is called stress vector [2]. The concentrated torque on a small but finite size surface figure can be interpreted similarly [2]:

$$
\int_{\Delta A} \mathbf{r} \times \boldsymbol{\sigma}_{\mathbf{n}}(x, y, z) y d A=\mathbf{M}_{\mathbf{n}, \triangle A}(x, y, z) .
$$

The equilibrium of elemental tetrahedron can be examined with the help of forces and torques interpreted on a plane figure. It can be verified with force equations that stresses form a second order tensor quantity, and it can be verified with torque equations that the stress tensor is symmetrical [2].

The equilibrium of the elemental cuboid can be examined with the help of forces and torques interpreted on a surface figure. The general form of force equations is

$$
\frac{\partial \sigma_{x i}}{\partial x}+\frac{\partial \sigma_{y i}}{\partial y}+\frac{\partial \sigma_{z i}}{\partial z}=0, \quad(i=x, y, z)
$$

which can be extended with body force and the inertia parameter [2], the torque equations are met due to the symmetry of the stress tensor.

Note: We have 6 dynamic variables, with three relationships (the equilibrium equations) among them. The dynamic description of the continuum is indefinite.

There are relationships and material equations between quantities characterising the kinematic and dynamic side and provide exactly as many equations as the number of variables in the system. 


\subsection{Possibilities and limitations of the continu- ous in physical space model}

The continuous model is based on many, primarily topological and metric assumptions (see [6,7]).

The model can describe the change in shape of the deformable solid body (locally the relative stretches and also locally the angle change between two curves), and the internal forces (stresses) distributed in the body.

The model cannot express the rotation of a point, the force acting upon a point, and the torque acting upon a point as a point has no dimensions and surfaces act upon each other rather than points. At the same time the model is suitable for expressing the rotation of a direction vector and the neighbourhood of a point as well as assigning force and torque to a small but finite size surface figure with any outward normal vector in the point.

The model only describes the change kinematically correctly in case of fixed atomic-molecular order or strained (unchanged topology) particles. Therefore e.g.: in case of atomic structure the strain of the crystalline grid is described accurately by the continuous model. It cannot describe the rearrangement of the atomic order accurately in case of plastic flow as the rearrangement is accompanied by the damage of the topologic order. Similarly, the model does not reveal the rearrangement of atoms in fluids, the collisions of atoms-molecules in gases and their changeovers either.

The model can only describe the collective movement of molecules. Therefore the model is suitable for describing acoustic vibrations not for the optical branch. It is capable of describing laminar flow in fluids and gases with adequate accuracy but no the cross diffusion.

The model is not suitable for determining force between atoms-molecules and particles as it can only interpret defined force and torque in the integrated sense assigned to the surface figures of a small but finite size.

The model does not reveal kinematic and dynamic parameters related to dimension of elements (atoms, molecule, particle) considered to be finite. Therefore, the values of forces between atoms and molecules constituting the matter cannot be determined in the model nor the values of rotation of particles and the torques occurring during rotation.

\section{Discrete in the physical space, con- tinuous in the phase space model}

\subsection{Description of the discrete in the physical space, continuous in the phase space model}

The matter is still considered to be the system of finite dimension elements in the physical space. (The data listed in the discrete model are known for the task; see the first paragraph of point 2.1.) We presume about this model that it is periodical and fixed. Because it is fixed, the behaviour of the medium can be characterised with the fact that the elements constituting the medium do not change places, they only move around their rest position at most. We presume in the study that in the grid points a mass point, a rigid body or a deformable solid body are located.
We assigned one state function to all elements collectively and not to each and every element in the phase space. We consider displacement as the example. Instead of the $\mathbf{u}\left(P\left(x_{i}, y_{j}, z_{k}\right)\right)$ displacement vectors assigned to the various $P\left(x_{i}, y_{j}, z_{k}\right)$ points, we consider one, the displacement vector field $\mathbf{u}(x, y, z)$. Consequently, instead of the $\mathbf{u}\left(P\left(x_{i}, y_{j}, z_{k}\right)\right)$,exact” solution, we consider the ,approximate" solution $\mathbf{u}(x, y, z)$. The „transition” is briefly marked:

$$
\mathbf{u}\left(P\left(x_{i}, y_{j}, z_{k}\right)\right) \Rightarrow \mathbf{u}(x, y, z) .
$$

In this case we have not yet made the theoretical description continuous in phase space. The mechanical relationships interpreted in discrete points are still interpreted in discrete points, but it is not written in the $\mathbf{u}\left(P\left(x_{i}, y_{j}, z_{k}\right)\right)$ form, but in the $\left.\mathbf{u}(x, y, z)\right|_{P\left(x_{i}, y_{j}, z_{k}\right)}$ form.

(There are the same numbers of variables in both forms.)

The next step of continuous model construction in the phase space is to find such continuous equations, which describe the mechanical state of the discrete system of the continuous kinematic $\mathbf{u}(x, y, z)$ and dynamic analogue with this variables, as well as the material relations. It has to be noticed that we approach the ,transition" analogue under (13) with one of the steps applied in the numerical method, the selection of approaching functions: the unknown functions are approached by the linear combination of known basis (functions), and we set up algebraic equations for determination of unknown real numbers (namely coefficients of the known basis functions). The next step of model construction in the phase space has to be analogue with the other step of numerical method: the distance of the exact and approximate solution has to be determined and this distance has to be minimised or made orthogonal for any (complete) vector of the space of the applied basis function. The numerical method applies to the approximate solution of the known equation. The task during the establishment of the continuous model in the phase space is to set up (continuous) state equation related to the state functions interpreted as continuous one in the phase space. As we do not have a continuous operator in the phase space, we cannot interpret the distance of the exact and approximate solution for the numerical method in an analogue way. Consequently, it is not sufficient to consider the state functions as continuous when creating a continuous in the phase space model, but the related state equations also have to be "selected" continuous. The selection of the equation cannot be arbitrary as it is to be applied to describe a given mechanical system. The only possibility is to apply analogy. Avoiding details, in order to create a continuous in the phase space model, the Lagrangefunction of the discrete system has to be made continuous as the example in (13):

$$
\begin{array}{r}
L\left(\mathbf{u}\left(x_{i}, y_{j}, z_{k}\right), \mathbf{F}\left(x_{i}, y_{j}, z_{k}\right), \ldots, P\left(x_{i}, y_{j}, z_{k}\right)\right) \Rightarrow \\
\Rightarrow L(\mathbf{u}(x, y, z), \boldsymbol{\sigma}(x, y, z), \ldots, P(x, y, z)) .
\end{array}
$$


Not the difference of the function values taken up in the discrete points but its continuous counterpart has to be included in the continuous Lagrange-function; applying the Taylor Series, for displacement:

$$
\mathbf{u}\left(P\left(x_{i}, y_{j}, z_{k}\right)\right)-\mathbf{u}\left(P\left(x_{i-1}, y_{j}, z_{k}\right)\right) \Rightarrow \partial \mathbf{u}(x, y, z) / \partial x .
$$

Interpreting displacement $(\mathbf{u})$, rotation $(\boldsymbol{\varphi})$ and the strain modus $(\boldsymbol{\varepsilon})$ as primary kinematic variable, the secondary kinematic variables are interpreted with the relationships below:

$$
\begin{aligned}
& \boldsymbol{\varepsilon}_{\mathbf{u}}(x, y, z) \Rightarrow \nabla \mathbf{u}(x, y, z), \\
& \boldsymbol{\varepsilon}_{\boldsymbol{\varphi}}(x, y, z) \Rightarrow \nabla \boldsymbol{\varphi}(x, y, z), \\
& \boldsymbol{\varepsilon}_{\boldsymbol{\varepsilon}}(x, y, z) \Rightarrow \nabla \boldsymbol{\varepsilon}(x, y, z) .
\end{aligned}
$$

Formally, to the analogy (13) of the discrete-continuous ,transition”, a $\mathbf{F}\left(P\left(x_{i}, y_{j}, z_{k}\right)\right) \Rightarrow \boldsymbol{\sigma}(x, y, z)$ type discrete-continuous ,transition" should be also applied in the case of internal forces. In practice, we consider the partial differential quotients according to kinematic variables of the Lagrange-function as (generalised) dynamic variables. For these, in the case of the $\mathbf{u}, \boldsymbol{\varphi}$ and $\boldsymbol{\varepsilon}$ variables

$$
\begin{gathered}
\boldsymbol{\sigma}_{\mathbf{u}}(x, y, z) \Rightarrow \partial L\left(\mathbf{u}(x, y, z), \boldsymbol{\varepsilon}_{\mathbf{u}}(x, y, z), \ldots, \mathbf{r}\right) / \partial \boldsymbol{\varepsilon}_{\mathbf{u}}, \\
\boldsymbol{\sigma}_{\varphi}(x, y, z) \Rightarrow \partial L\left(\varphi(x, y, z), \boldsymbol{\varepsilon}_{\varphi}(x, y, z), \ldots, \mathbf{r}\right) / \partial \boldsymbol{\varepsilon}_{\varphi}, \\
\boldsymbol{\sigma}_{\varepsilon}(x, y, z) \Rightarrow \partial L\left(\boldsymbol{\varepsilon}(x, y, z), \boldsymbol{\varepsilon}_{\varepsilon}(x, y, z), \ldots, \mathbf{r}\right) / \partial \boldsymbol{\varepsilon}_{\boldsymbol{\varepsilon}}
\end{gathered}
$$

relationships can be set up. The generalised Hooke's law for the generalised continuum can be set up in the form:

$$
\left[\begin{array}{c}
\sigma_{u} \\
\sigma_{\varphi} \\
\sigma_{\varepsilon}
\end{array}\right]=\left[\begin{array}{lll}
C_{u u} & C_{u \varphi} & C_{u \varepsilon} \\
C_{\varphi u} & C_{\varphi \varphi} & C_{\varphi \varepsilon} \\
C_{\varepsilon u} & C_{\varepsilon \varphi} & C_{\varepsilon \varepsilon}
\end{array}\right] \cdot\left[\begin{array}{c}
\varepsilon_{u} \\
\varepsilon_{\varphi} \\
\varepsilon_{\varepsilon}
\end{array}\right] .
$$

Both in (19-21), and (22) we set out from the fact that the material equations, namely relations between the generalised strains and the generalised internal forces, are known.

The state equations (the description limited to equilibrium), in accordance with the method of the model creation, are provided with by determining the maxima of the $L$ functional describing the state. Formally, the following three equations are set up for variables $\mathbf{u}, \boldsymbol{\varphi}$ and $\varepsilon$ :

$$
\begin{aligned}
& \partial L\left(\mathbf{u}(x, y, z), \boldsymbol{\varepsilon}_{\mathbf{u}}(x, y, z), \ldots, P(x, y, z)\right) / \partial \boldsymbol{\varepsilon}_{\mathbf{u}}= \\
& =\partial L\left(\mathbf{u}(x, y, z), \boldsymbol{\varepsilon}_{\mathbf{u}}(x, y, z), \ldots, P(x, y, z)\right) / \partial \mathbf{u}, \\
& \partial L\left(\boldsymbol{\varphi}(x, y, z), \boldsymbol{\varepsilon}_{\boldsymbol{\varphi}}(x, y, z), \ldots, P(x, y, z)\right) / \partial \boldsymbol{\varepsilon}_{\boldsymbol{\varphi}}= \\
& =\partial L\left(\boldsymbol{\varphi}(x, y, z), \boldsymbol{\varepsilon}_{\boldsymbol{\varphi}}(x, y, z), \ldots, P(x, y, z)\right) / \partial \boldsymbol{\varphi}, \\
& \partial L\left(\boldsymbol{\varepsilon}(x, y, z), \boldsymbol{\varepsilon}_{\varepsilon}(x, y, z), \ldots, P(x, y, z)\right) / \partial \boldsymbol{\varepsilon}_{\boldsymbol{\varepsilon}}= \\
& =\partial L\left(\boldsymbol{\varepsilon}(x, y, z), \boldsymbol{\varepsilon}_{\boldsymbol{\varepsilon}}(x, y, z), \ldots, P(x, y, z)\right) / \partial \boldsymbol{\varepsilon} .
\end{aligned}
$$

Finally, the functional - the Lagrange-function of the system -, which describes the state of the system also has to be given. The continuous Lagrange-function in the phase space can only be set up based on analogy. Regularly, we set out from the fact that kinetic energy is proportional to the square of velocity, while elastic energy is proportional to the square of the strain. Taking this into consideration, based on analogy, the Lagrange-function can be constructed. The state equations of the system for variables $\mathbf{u}, \boldsymbol{\varphi}$ and $\boldsymbol{\varepsilon}$ can be set up in the following operator form:

$$
\begin{gathered}
I_{\mathbf{u u}} \ddot{\mathbf{u}}_{\mathbf{u}}-\nabla\left(\mathbf{C}_{\mathbf{u u}} \nabla \mathbf{u}\right)-\nabla\left(\mathbf{C}_{\mathbf{u} \varphi} \nabla \boldsymbol{\varphi}\right)-\nabla\left(\mathbf{C}_{\mathbf{u} \varepsilon} \nabla \boldsymbol{\varepsilon}_{\mathbf{u}}\right)=\mathbf{Q}_{\mathbf{u}} . \\
I_{\varphi \varphi} \ddot{\boldsymbol{\varphi}}_{\varphi}-\nabla\left(\mathbf{C}_{\varphi \mathbf{u}} \nabla \mathbf{u}\right)-\nabla\left(\mathbf{C}_{\boldsymbol{\varphi} \varphi} \nabla \boldsymbol{\varphi}\right)-\nabla\left(\mathbf{C}_{\varphi \varepsilon} \nabla \boldsymbol{\varepsilon}_{\varphi}\right)=\mathbf{Q}_{\varphi} . \\
I_{\varepsilon \varepsilon} \ddot{\boldsymbol{\varepsilon}}_{\varepsilon}-\nabla\left(\mathbf{C}_{\varepsilon \mathbf{u}} \nabla \mathbf{u}\right)-\nabla\left(\mathbf{C}_{\varepsilon \varphi} \nabla \boldsymbol{\varphi}\right)-\nabla\left(\mathbf{C}_{\varepsilon \varepsilon} \nabla \boldsymbol{\varepsilon}_{\varepsilon}\right)=\mathbf{Q}_{\varepsilon} .
\end{gathered}
$$

The $I_{i j}$ quantities are inertias of the ,spread" matter in the generalised continuum versus the displacement, rotation and strain. The analogy is perfect with the motion equations of the classic continuum because the formulas were compiled according to its formalism. Such conditions from mathematical aspects exist, which ensure the correctness of the targeted boundary values ( [8]).

\subsection{Characterisation of discrete in the physical space, continuous in the phase space model}

The continuous in the phase space model is constructed based on analogy. The continuous, secondary kinematic variables, the continuous Lagrange-function of the system and the continuous internal forces are interpreted based on analogy. Following this, we set up the equations describing the state of the system based on the known mathematical algorithms. A peculiarity of the model is that no such experiments exist, which would enable direct measurement of material constants. While in the case of classic continuum, from the differential geometric description it can be concluded such experimental arrangement (uniaxial pull and shear), which connects the six strains of an elemental cube to stress assigned to three surfaces of the elemental cube can be interpreted, in the case of the generalised continuum no such geometrical shape exists, where the dynamic variables could be clearly assigned to its displacements. In case of generalised continuums not simply single-parameter experiments are required for the verification of the model. Firstly, the introduced kinematic and dynamic variables have to be interpreted, secondly, such experimental arrangements have to be prepared, where these phenomena can be detected, measured and the experiments have to be carried out, thirdly, the theoretical tasks regarding the targeted experimental arrangement have to be set and have to be solved. Finally, the experimental results have to be compared with the theoretically determined results. As many experimental states have to be interpreted as the number of material constants exist in the theory. If these material constants are determined in accordance with the experiments, that is to say the parameters in the theory are 
fitted to the measured results then the theory can be used for the task to be solved.

The model describes the state of points located on grid points; the values of continuous functions taken up on grid points have mathematical and mechanical senses.

The model can be created preferably when the mechanical relationship among elements located on grid points is known.

\section{Summary}

We overviewed three possibilities of modelling the mechanical behaviour of media. The first possibility, is that the motion of each and every mass particle constituting the medium described by an individual equation. The functions describing the state of the medium obtain a value in the location of mass particles characterised by it. The system is discrete from this aspect. This possibility can be restricted to the concurrent examination of a few thousand or ten thousand mass particles; it is not suitable for the examination of millions of particles or at atomic level for the concurrent examination of the scale of $10^{23}$ gas molecules present in one cubic decimetre. As a second possibility, the application of some continuous functions has to be mentioned instead of the discrete domain functions. This in the first approach means that the state of the system described by a few functions interpreted in the continuous domain of some continuum consisting of many points including independent points instead of functions interpreted in a finite number of independent points. Two such spaces can be identified. One is the physical space, where the elements of the medium move, while the other is the phase space, where the state of the elements of the medium are mathematically characterised. Therefore, the second possibility is to consider a region of the Euclidean space (which has continuum cardinality) instead of the examined finite number of particles, and we extend the mechanical relationships related to the particles to this domain. In this model construction, we „spread" all mass present in points by default in one continuous region of the Euclidean space modelling the physical space, we have no mass particles but a medium with continuous distribution exists, along with this the functions characterising the system are continuous functions interpreted on a continuum. This model creation is based on differential geometry. This method leads to the term of classic continuum and as such only one exists. The third possibility is to keep the finite number of many points with their independent, unique size, characteristics as models, at the same time we embed them into the finite region of the Euclidean space with the help of their reference points, then we interpret the continuous functions describing the system on this range and extend relationships related to the discrete mechanical system for these continuous domain functions. In this model construction, we ,spread" the functions describing the states in the phase space by default: continuous domain (con tinuous) functions are included instead of discrete domain functions. We create the continuous domain Lagrange-function of the system and determine the state indicators and state equations for continuous functions. This model creation applies steps of the numerical method (selection of basic functions, creation of error principle). This method is suitable for describing fixed, periodical structure rigid bodies and leads to the generalised continuums. The generalised continuum, contrary to its name, is not continuous but a discrete system with typical discrete states (e.g.: optical vibration branch of atoms), which do not exist in the classic continuum. Many generalised continuums exist, these form a hierarchy.

\section{References}

[1] Love, A.E.H. A Treatise on the Mathematical Theory of Elasticity. Fourth ed. Cambridge, At the University Press (1927)

[2] Lur'e, A.I.: Theory of Elasticity. In Russian. Nauka, M. (1970)

[3] Lámer G.: Notes on the Theory of Large Displacement with Small Strain = Periodica Politechnica 29 (1-2), pp. 53-65 (1985)

[4] Lámer G.: Mathematical Foundations of the Theories of Perfect Elastic Shells and Rods Underdoing Large Displacement with Small Strains. PhD Dissertation. (A kis alakváltozások mellett nagy elmozdulásokat végzö tökéletesen rugalmas héjak és rudak elméleteinek matematikai alapjai) Budapest (1990)

[5] Lámer G.: On the Kinematics of the Continuum Underdoing Large Displacement with Small Strains. (Kis alakváltozások mellett nagy elmozdulást végző kontinuum kinematikájáról) $=$ Épités, Épitészettudomány XXIII (1-2), pp. $35-59$ (199293)

[6] Lámer G.: Opportunity and Limit of Applications of the Topological Tools in the Mechanical Models for the Media. (Topológiai eszközök alkalmazásának lehetőségei és korlátai a közegek mechanikai modellezésében). Proceedings of XII. MAMEK (Miskolc, 2015. aug. 25-27.) Ed.: Baksa A. - Bertóti E. - Szirbik S. paper 211. p. 13 (2015)

[7] Lámer G.: Opportunity and Limit of Applications of the Metrical Tools in the Mechanical Models for the Media. (Metrikus eszközök alkalmazásának lehetőségei és korlátai a közegek mechanikai modellezésében. Proceedings XII. MAMEK (Miskolc, 2015. aug. hó 25-27.) Ed.: Baksa A. - Bertóti E. - Szirbik S. paper 326. p. 13 (2015)

[8] Kunin, I.A.: Theory of Elastic Media with Microstructure. In Russian. Nauka, M. (1975) 